\title{
Grupos de encuentro: desarrollo de habilidades para la humanización del cuidado, a partir de la experiencia de aprendizaje de estudiantes de enfermería
}

\author{
Claudia Moya ${ }^{1}$ \\ Claudio Pulgar² \\ Aaron Trajtmann ${ }^{3}$
}

Recibido 08 de octubre de 2019 - Aceptado 31 de octubre 2019

\begin{abstract}
RESUMEN
Ser profesional de enfermería implica cualidades que permitan gestionar integralmente el cuidado de personas que están en situación de enfermedad. Los grupos de encuentro, como experiencia de aprendizaje, permiten a los estudiantes reconocerse y reconocer a otro en su proceso de salud-enfermedad. ¿Qué aprendizajes descubro para mi vida personal y profesional con las experiencias del curso?, fue la pregunta que se hizo el equipo de salud mental de una universidad particular de Santiago. Un observador externo fue testigo de la estrategia educativa de grupos de encuentro y, mediante notas de campo, estableció palabras, frases y argumentos que otorgaban significado al proceso experiencial vivenciado. De este modo, se estructuró una pauta para medir la percepción de los estudiantes respecto de la asignatura y aprendizajes obtenidos, la que fue aplicada al finalizar la experiencia. Mediante un cuestionario online, de forma anónima, II 2 estudiantes respondieron la encuesta. Los resultados son muy esclarecedores: un alto porcentaje valoró positivamente los contenidos y las experiencias vividas, afirmando que la asignatura aporta a sus proyectos de vida, reforzando además la dimensión vocacional. Los grupos de encuentro potencian y desarrollan las habilidades interpersonales y de acompañamiento emocional, contribuyen a que los estudiantes puedan reconocer sus emociones, utilizar el cuerpo como indicador de lo que les sucede, desarrollar la autoconciencia, vivenciar el presente e integrar a la persona en el profesional de enfermería, fortaleciendo la capacidad de cuidar a otros, elementos claves de la humanización del cuidado.
\end{abstract}

Palabras clave: Grupos de encuentro, desarrollo de habilidades, experiencia de aprendizaje, humanización del cuidado.

I Enfermera, Psicóloga, Magíster, Académico, Universidad San Sebastián. Contacto: claudia.moya@uss.cl.https://orcid.org/0000-000I-9758-I224

2 Trabajador Social, Magíster, Académico, Universidad San Sebastián. Contacto: cpulgarcartagena@gmail.com.https://orcid.org/0000-0003-I22I-9678

3 Psicólogo, Magíster, Académico, Universidad San Sebastián. Contacto: aaron.trajtmann@ gmail.com, https://orcid.org/0000-0002-597I-I056 


\title{
Encounter Groups: Development of Skills for the Humanization of Healthcare, Based on Nursing Students' Learning Experience
}

\begin{abstract}
The nursing profession implies qualities that enable the integral management of healthcare for the sick. Encounter groups, as a learning experience, allow students to recognize themselves and others in their health-sickness process. What have I learned for my personal and professional life from the experiences in this course?, was the question posed by the mental health team at a private university in Santiago. An external observer was witness to the educational strategy of encounter groups and, through field notes, was able to establish words, phrases and arguments that gave greater meaning to the experiential process. This was used to structure an instrument to measure student perception of the course and the learning outcomes, which was applied at the end of the experience. Through an anonymous online questionnaire, II2 students responded to the survey. The results are very enlightening: a high percentage rated the contents and experiences positively, stating that the course contributed to their life project, while reinforcing their vocation. The encounter groups strengthen and develop interpersonal and emotional support skills, and help student recognize their emotions, use their body as an indicator of what they are feeling, develop self-awareness, live in the present and integrate the person within the nursing professional, thus enhancing their ability to care for others, all key elements of the humanization of healthcare.
\end{abstract}

Key words: Encounter groups, skills development, learning experience, humanization of healthcare.

\section{Introducción}

En la formación profesional de Enfermería, los estudiantes requieren constantemente herramientas para acercarse al dolor, la angustia y el sufrimiento; gestionando el cuidado de personas que están en situación de enfermedad, minusvalía o agonía. Por lo que entender los procesos educativos y de acompañamiento a los futuros profesionales se hace fundamental e implica una actitud de cuestionamiento y revisión sistemática de las prácticas docentes. Con base en esta revisión, la asignatura de Comunicación e Interacción Humana (CIH), fundamentada en el enfoque centrado en la persona de Carl Rogers (1973), busca acompañar el proceso de desarrollo de habilidades interpersonales y de apoyo emocional en los estudiantes de Enfermería. Trabajar desde lo afectivo, no es tarea fácil, como dice Kepner 1987, (citado en Almada 2015), mostrar las emociones puede ser difícil si, al tratar de establecer contacto con alguien, se es criticado o rechazado, por lo que se vuelve un riesgo mostrar la propia necesidad de amor. Durante el transcurso de la asignatura, 
las experiencias vividas y narradas por los estudiantes permiten responder a la pregunta: ¿qué aprendizajes descubro para mi vida personal y profesional con las experiencias del curso? Cada estudiante comprende que las experiencias vividas en la asignatura son las propias de quien descubre lo importante de tomar conciencia de sí mismo, logrando una comprensión profunda del valor del autoconocimiento; valora la experiencia de saber lo que siente, escuchando lo que pasa con su cuerpo; comprendiendo que sus reacciones pueden interrumpir su relación con los otros, por no saber cómo se está construyendo ese mundo relacional, focalizándose en las dinámicas de interacción ocurridas en el aquí y ahora. Conocer los aprendizajes y significados que a cada estudiante le hacen sentido en el ámbito personal y profesional, es el alcance de esta investigación en educación.

En medio de la complejidad de nuestra sociedad actual, se espera que un enfermero, más allá de poseer conocimientos técnicos, esté preparado para enfrentar un mundo cambiante desde una mirada profesional, sin dejar de lado su propia persona. Esto hace necesario utilizar nuevas prácticas en educación que permitan acceder al ser humano que cuida, lo que muchas veces se contrapone con las lógicas establecidas por la mayoría de las estructuras educacionales.

\section{Marco referencial}

La Organización Mundial de la Salud (20I4) declara que uno de los principales indicadores de desarrollo, siendo además un derecho fundamental de todo ser humano, es gozar el grado máximo de salud que se pueda lograr. Recordando que la salud es un estado de completo bienestar físico, mental y social, y no solamente la ausencia de afecciones o enfermedades.

En ese contexto se enmarca la formación de los diversos profesionales de la salud, entre ellos las enfermeras y enfermeros, quienes, en su proceso de formación, reciben diversos aprendizajes para asegurar este mencionado derecho humano.

Un completo grupo de aprendizajes en esta línea es recibido, en el contexto de la educación universitaria, por enfermeras y enfermos en una universidad privada de Santiago de Chile, y se enmarca en 
los 1lamados "grupos de encuentro" (Rogers, I973), los cuales se desarrollan en la asignatura de Comunicación e Interacción Humana y se basan en los planteamientos de la psicología humanista.

\section{La educación desde el humanismo}

El humanismo, en el contexto del aprendizaje, es una corriente que señala la importancia de la dimensión socioafectiva de los individuos, de las relaciones interpersonales y de los valores en los escenarios educativos, terapéuticos y sociales como factores determinantes en el aprendizaje. En ese contexto, Carl Roger propone una educación democrática centrada en la persona, la cual consiste en otorgar la responsabilidad de la educación al estudiante (Rogers, I969).

La perspectiva humanista reconoce al ser humano con libertad de elección y no bajo la influencia de fuerzas inconscientes o refuerzo externo. Las personas son libres de elegir sus objetivos y tratan de sortear las limitaciones individuales para desarrollar sus capacidades mentales. Rogers cree que los estudiantes pueden lograr resultados de aprendizaje óptimos si son tratados por personas significativas en su entorno, con respeto incondicional, autenticidad y empatía, y si se les permite asumir la plena responsabilidad de su propio aprendizaje. La perspectiva humanista puede caracterizarse como principalmente preocupada por las necesidades individuales, valores, motivaciones, etc. (Guey, Cheng \& Shibata, 2010).

Desde esa perspectiva, la educación humanista consistiría en facilitar en el individuo el desarrollo de sus propias potencialidades o, parafraseando a Carl Rogers, crear un ambiente propicio para que el educando "pueda ser lo que es". Por tanto, se aprende (comprende) de la experiencia, siendo este aprendizaje un proceso activo, afectivo y emocional, en el que participa el organismo como totalidad, considerando a las personas como fines en sí mismas y no como simples instrumentos para conseguir otros objetivos (Capo, I986). Como carrera de la salud, es relevante en el quehacer de Enfermería la necesidad de involucrar la emoción, dado que cuidar a un otro tendría un impacto en sí mismo en el sujeto que cuida, influyendo en su práctica profesional. Así, el autoconocimiento es una faceta 
importante: significa reconocer quién es y qué hacen las enfermeras/ os (Rasheed, Younas \& Sundus, 2019; Walter, Davis \& Glass, I999).

Es necesario cuestionar el paradigma utilizado para enseñar y facilitar el autoconocimiento. La transferencia didáctica tradicional del conocimiento, que tiende a ser el pilar de muchos programas educativos, incluyendo el de Enfermería, parece ser inconsistente, con un proceso subjetivo e intrapersonal que no puede ser forzado. En este contexto, toma sentido que se fomente la autoconciencia de mejor manera, en un paradigma de enseñanza-aprendizaje que considere el procesamiento cognitivo y afectivo desde múltiples perspectivas, en lugar de la transmisión a través de la memorización (Eckroth-Bucher, 2010).

La educación centrada en la persona confiere la responsabilidad de la educación al mismo educando; su objetivo radica en crear las condiciones favorables que faciliten el aprendizaje y que liberen las capacidades de autoaprendizaje desde una perspectiva globalizante de lo intelectual-emocional. Se pretende ayudar a los estudiantes a que se conviertan en seres con iniciativa, responsabilidad y autodeterminación; que sepan solucionar creativamente los problemas y puedan adaptarse con flexibilidad a las nuevas situaciones, propias de una sociedad en continuo cambio. Se tiene la mira puesta en conseguir personas que sepan colaborar solidariamente con los demás, sin que por ello deban renunciar a su individualidad (Capo, 1986). Para el educador humanista, la tarea principal consiste en ayudar a la persona a encontrar lo que tiene en sí misma; no reforzarla o formarla de un modo predeterminado que alguien ha decidido de antemano, a priori (Maslow, I968).

\section{Tensión entre paradigmas}

Las carreras de la salud contrastan muchas veces con el paradigma humanista, generando una tensión que surge de la mirada científica objetivable. En este contexto, la validez de la perspectiva humanista en el aprendizaje es difícil de examinar, en parte porque las ideas humanísticas son filosóficas y abstractas; por lo tanto, no son fácilmente objetivables (Aliakbari, Parvin, Heidari \& Haghani, 2015), necesitando 
más investigación empírica para aplicar los conocimientos teóricos disponibles en la práctica (Rasheed et al., 2019).

Siguiendo a Glass y Glass (I98I), nuestra cultura:

Está muy orientada hacia los resultados, y los resultados de la educación humanística no solo son difíciles de evaluar, sino que, a menudo, tardan en aparecer. El proceso del aprendizaje autodirigido también puede parecer una pérdida de tiempo. Estos elementos crean nerviosismo en nuestra cultura, donde perder tiempo equivale a perder la oportunidad para el logro del producto, lo cual es sinónimo de malgastar el dinero. Esto es un problema para todos los que creemos en la educación autodirigida, en la educación como proceso, y en el aprendizaje permanente. (p. 77).

Esto exige un gran desafío para los educadores, puesto que se contrapone con las lógicas establecidas por la mayoría de las estructuras educacionales, debido a que un educador humanista debe permitir que los estudiantes puedan tener voz e ideas propias (a veces contrarias a sus profesores); los maestros deben estar seguros de sí mismos y sin la necesidad imperiosa de mantener el rol fijo de "saber todo" (Capo, I986).

Humberto Maturana destaca la importancia que tienen las emociones en el juego de educar, reconociendo la integralidad del ser humano, más allá de la razón. Al respecto, señala que todos los dominios racionales que tenemos a la mano como seres humanos, cualquiera que sea el dominio operacional en que ocurren las acciones que los constituyen, tienen un fundamento emocional, debido a que están constituidos por la aplicación consistente de algunas premisas básicas, directa o indirectamente aceptadas sin justificación racional, sino como resultado de alguna preferencia (Maturana, 200I).

Educar es enriquecer la capacidad de acción y de reflexión del ser aprendiz, es desarrollarse en comunión con otros seres. Desarrollarse en la biología del amor, que nos muestra que "el ser vivo es una unidad dinámica del SER y del HACER” (Maturana \& Nisis de Rezepka, 1997). Así, la educación es, para esos autores, un proceso de transformación en la convivencia, en la que el aprendiz se transforma 
junto con los profesores y con los demás compañeros con los cuales convive en su espacio educacional, tanto en lo que se refiere a las transformaciones en la dimensión explícita o consciente, como en la dimensión implícita o inconsciente

Los ambientes educacionales deben constituirse en espacios de acción y reflexión, ambos fundamentados en la emoción, recordando que la reflexión se constituye también en un acto de desapego, al admitir que aquello que pensamos, deseamos, opinamos, analizamos y hacemos, puede ser pensado, analizado, observado, refutado o construido de diferentes maneras, a partir de un análisis más reflexivo (Maturana \& Nisis de Rezepka, 1997)

\section{Grupos de encuentro}

Kurt Lewin, en 1947, promovió la idea de que la formación en relaciones humanas constituía un tipo de educación importante pero descuidado en la sociedad moderna. Posterior a Lewin, aparecen los grupos $\mathrm{T}$ (donde $\mathrm{T}$ designa training, capacitación) que eran grupos de capacitación en habilidades vinculadas con las relaciones humanas (Rogers, 1973). Algunos años después, Carl Rogers emplea el término para designar a grupos de trabajo que tienen como meta el crecimiento personal y la realización de las potencialidades humanas (Schutz, 1973).

Quienes han participado en estos grupos relatan los efectos sorprendentes que han surtido en su matrimonio, su carrera o los sentimientos que tenían acerca de sí mismos, también acerca de la forma en que cambió su vida a partir de entonces. El encuentro implica una relación franca con los demás, conciencia de uno mismo y total unidad del sí mismo. Cuando se mira por encima a la gente resaltan sus diferencias: negros y blancos, hombres y mujeres, seres agresivos y pasivos, intelectuales y emocionales, alegres y tristes, radicales $y$ reaccionarios. Pero a medida que comprendemos a los demás, las diferencias desaparecen y en su lugar surge la unicidad humana: las mismas necesidades, los mismos temores, las mismas luchas y deseos.

Los grupos de encuentro ayudan a la gente a trascender las distintas formas en que cada uno aborda su vida, y a mirar el entorno para 
descubrir entonces que todos tratan de hacer frente al mismo problema, solo que con distintos procedimientos (Schutz, 1973). En grupos como estos el individuo llega a obtener un conocimiento de sí mismo — y de cada uno de los demás - más completo que el que logra en sus relaciones sociales o de trabajo habituales. Puede conocer de manera profunda a los otros miembros y su propio ser interior, el ser que, de otro modo, tiende a ocultarse detrás de su fachada (Rogers, 1973).

El concepto que tenemos de nosotros mismos deriva en gran medida de nuestras relaciones con el resto de la gente. Intercambiamos con ella diversas mercancías y debemos hacer diversos ajustes en este intercambio (Schutz, I973). Las creencias sobre quiénes somos en el mundo, nuestros miedos, esperanzas y expectativas de lo que somos y de lo que podemos ser en el futuro, son aspectos fundamentales del concepto de uno mismo que influyen poderosamente en nuestras acciones (Stein, 1995). Existe también evidencia de la importancia que debe tener para los enfermeros comprender la conducta verbal y no verbal en la interacción con los pacientes (Egan, 2010; Jack \& Smith, 2007; Parveen, 20I5), siendo necesario aprender a reaccionar ante distintas situaciones, respetando las diferencias socioculturales (Parveen, 2015).

Aumentar los niveles de autoconocimiento enriquece las interacciones que el sujeto puede tener en distintos ámbitos de su experiencia. De alguna manera permite descubrir nuevos caminos, tanto en las relaciones con otros como en la vida (Engin \& Cam, 2009; Jack \& Smith, 2007; Rowe, 1999). De igual forma, la creación de un entorno interpersonal que sana solo es posible si los enferemeros utilizan las habilidades de autoconciencia como guía en el proceso de comunicación. Por el contrario, carecer de adecuados niveles de conciencia de sí mismo genera gastos elevados de energía, reprimiendo conflictos personales, la cual podría utilizarse para prestar atención al presente y aprender vivencialmente de sí mismo (Eckroth-Bucher, 2010; Scheick, 20II).

Quien realiza un rol de ayuda, menciona Rogers (2007), debe ser congruente, genuino e integrado. Es decir, en la interacción debe mostrarse profundamente a sí mismo, con su experiencia real. Siendo lo opuesto a presentar una fachada o un modelo que exhibe integridad en cada aspecto de su vida. Es suficiente que él sea exactamente sí mismo en la relación, que sea lo que realmente es en ese momento. 
La autoconciencia puede ser una herramienta importante para mejorar la interacción enfermero-paciente y debería ser una parte integral de los programas de educación en enfermería. A medida que los enfermeros se vuelvan más conscientes de sí mismos y actúen sobre cualquier debilidad percibida, el aspecto terapéutico de la enfermería mejorará (Rowe, I999).

\section{La subjetividad de las emociones}

A diferencia de lo que se pueda creer desde la generalidad, las emociones son mucho más que una cosa "sentimental" o "romántica", sino que tienen raíces biológicas, psicológicas, corporales, sociales y por tanto científicas, y se configuran como un motor esencial para la articulación y desarrollo vital del ser humano.

Susana Bloch define las emociones como un "estado funcional dinámico de todo el organismo, gatillado por un estímulo interno o externo que implica simultáneamente la activación de un grupo de órganos efectores y una experiencia subjetiva” (Bloch \& Lemeingnan, 1992).

En ese contexto, podemos saber, incluso por sentido común o por experiencia, que ya sea que la situación emocional desencadenante venga del mundo externo o interno, la activación subjetiva que resulta está generalmente acompañada por elementos somáticos, tales como modificaciones de la expresión facial, en la dirección de la mirada, de la postura corporal, todos estos componentes expresivos de una emoción. A su vez, se acompaña por ciertos elementos fisiológicos, como cambios en las funciones viscerales, aumento de la frecuencia cardíaca, contracciones estomacales, cambios en la frecuencia respiratoria, rubor, palidez, aun cuando no siempre estemos conscientes de estas modificaciones.

Con esto en la base, es precisamente aquella experiencia subjetiva mencionada en la definición, la que es interna, personal afectiva y vivencial (Bloch \& Lemeingnan, 1992), y por tanto se vuelve particular a la historia de cada ser humano, historia que, desde la enfermería, se presenta frente a la acción profesional y para cuyo abordaje se debe contar con herramientas que permitan conocerla, interpretarla, incorporarla y abordarla en la atención en salud. 


\section{Tríada: cuerpo, mente y emoción}

Muchas veces, como seres humanos, nos olvidamos con facilidad de que el cuerpo, la mente y la emoción tienen un vínculo estrecho $y$, por tanto, se configuran como una unidad. En palabras de Schutz, el hombre es de hecho una unidad entre el cuerpo, la mente, los sentimientos, la conducta interpersonal y el espíritu, todas manifestaciones de una esencia única (Schutz, 1973).

Bajo esta premisa, en la medida que más consciente se está desde cada arista de esta unidad, más posible es administrar y hacerse responsable de las decisiones de manera consciente.

Para Schutz, el cuerpo juega un papel principal, puesto que, al saber lo que dice el cuerpo, es posible conocer los sentimientos más profundos, pudiendo elegir con libertad: "Con un conocimiento completo de mí mismo, puedo determinar mi vida" (Schutz, I973, p. I0). El autor plantea la certeza de que las afecciones y dolores en distintos lugares del cuerpo pueden ser signos de conflictos no resueltos que no están conscientes en la mente y que, por ende, son expresados por el cuerpo.

Desde la perspectiva de la asignatura de Comunicación e Interacción Humana, a ese fenómeno se le llama "proceso psíquico", el cual se inicia reconociendo la emoción y su correlato corporal, para luego tomar conciencia de lo que aquello significa hoy de manera reflexiva.

La importancia que el cuerpo tiene, por tanto, es que se transforma en un catalizador que sirve como indicador de lo que pasa a nivel interno, de las emociones que las vivencias pueden hacer aflorar, pudiendo establecer cambios si es que esta conciencia corporal permite un darse cuenta, que abra esas posibilidades de conciencia de sí.

En esa línea, Schutz habla de la importancia de trazarse un mapa de conciencia del cuerpo de cada individuo (Schutz, I973), puesto que cada uno, de manera particular, tendrá manifestaciones y correlatos corporales de acuerdo con su propia historia, su propias emociones y su propia forma de vivir las experiencias vitales, siendo la conciencia del cuerpo una herramienta importante para el trabajo personal y el desarrollo emocional. 


\section{Humanización del cuidado}

Los profesionales de la salud, al tomar contacto con la persona enferma, pueden percibir que la enfermedad ocasiona una alteración global en el sujeto y, de modo significativo, en el mundo afectivo y relacional. A esto se agrega que la condición de estar hospitalizado es un agravante que intensifica sentimientos de impotencia, dependencia, carencia, e incluso de despersonalización. El sujeto deja de ser como era y se torna paciente. En este contexto, toma contacto con la finitud, lo que trae una nueva conciencia, los valores y prioridades cambian, surgiendo interrogantes sobre el sentido de la existencia. La persona, frente a esta situación límite, experimenta una radical vulnerabilidad, siendo las acciones de cuidado una manera de ayudar al ser en estas circunstancias. Para ello es necesario estar preparados para ofrecer un cuidado que proporcione confort, no solo físico sino también espiritual. Además, considerar al otro como ser humano y no como objeto (Waldow, 20I4).

Existen elementos contextuales en la atención de salud que ponen en riesgo la pérdida de los atributos que hacen del cuidado una cualidad humana, desapareciendo la dignidad humana propia de la relación entre el profesional de salud y el paciente (Álvarez \& Román de Cisneros, 2008; Ceballos, 2010; Landman Navarro et al., 2016; Poblete Troncoso \& Valenzuela Suazo, 2007). Para la enfermería, es prioritario entregar un cuidado humanizado, siendo una profesión que requiere tener como base el autoconocimiento, además de un continuo dominio y reflexión sobre problemas de interacción humana desde un punto de vista ético, social y político. Esto requiere de conocimientos; se debe saber trabajar con seres humanos, de lo contrario, a los estresores laborales, presentes en los ambientes de trabajo, se agregan problemas de salud en los profesionales, tanto físicos como mentales, afectando la labor de cuidar. Todo esto resulta en que la entrega de cuidados resulta de menor calidad, no solo en lo procedimental, sino en el trato con el otro, pudiendo explicar la deshumanización que se genera en el cuidado (Ramos Guajardo \& Ceballos Vásquez, 2018).

No se debe olvidar que la percepción de cuidado humanizado es una dimensión de la calidad de los servicios de salud, definida como la experiencia advertida y expresada por el usuario que, en muchas 
ocasiones, no se relaciona con aspectos científicos o tecnológicos, sino con necesidades de afecto, comprensión, protección, bienestar, ocio e información (Romero Massa, Contreras Méndez \& Mondaca Serrano, 2016).

\section{Metodología}

El equipo de salud mental de Santiago de una Escuela de Enfermería en una universidad particular, solicitó la cooperación de la Dirección de Efectividad Educativa e Innovación para construir un proyecto de observación por el que se pudiera evidenciar la estrecha relación entre la asignatura de Comunicación e Interacción Humana y sus aportes a la dimensión vocacional y profesional de sus estudiantes.

El proyecto consistió en establecer una observación sistemática a la asignatura, impartida en el segundo año de la carrera, que utiliza la metodología de grupos de encuentro, en la que aproximadamente I5 estudiantes, sentados en círculo y junto a un facilitador, se reúnen una vez por semana (dos módulos continuos) con el fin de estimular el autoconocimiento y la comunicación entre ellos. Un observador externo, durante I0 sesiones, mediante notas de campo, estableció elementos (palabras, frases, argumentos) que otorgaban significado al proceso vivencial/experiencial de las sesiones grupales. Los elementos de significación descubiertos fueron contrastados con el perfil de egreso, lo que permitió abrir la discusión sobre la pertinencia de la metodología utilizada en esta asignatura. La base de este proyecto se fundamenta en que las habilidades interpersonales para un profesional de enfermería se vuelven vitales para el bienestar de sí mismo y de sus pacientes. Es decir, una habilidad como la empatía no se puede enseñar a través de una clase magistral, sino que debe ser aprendida en un contexto experiencial, mediante una vivencia. El pensamiento no resuelve los problemas, porque el problema no se origina con un pensamiento sino con una experiencia, con una vivencia, con una palabra o con el impacto de una presencia que nos marca (Borja, 1995).

De este modo, se estructuró una pauta para medir la percepción en la opinión de los estudiantes respecto de la asignatura. Los 
elementos consultados dicen relación con contenidos, conceptos trabajados en clases y la pertinencia de la asignatura en el proyecto vocacional de cada estudiante. Esta pauta fue aplicada al finalizar la asignatura, cuando I 12 estudiantes respondieron de forma anónima un cuestionario online de Google. Al inicio del cuestionario se les solicitó la autorización para utilizar los resultados en procesos de investigación en educación.

\section{Resultados}

La encuesta, realizada online, arrojó los siguientes resultados:

Grafico $\mathrm{n}^{\circ} \mathrm{I}$. Es una herramienta importante aprender a escuchar mi cuerpo, como un lenguaje que me habla de mí.

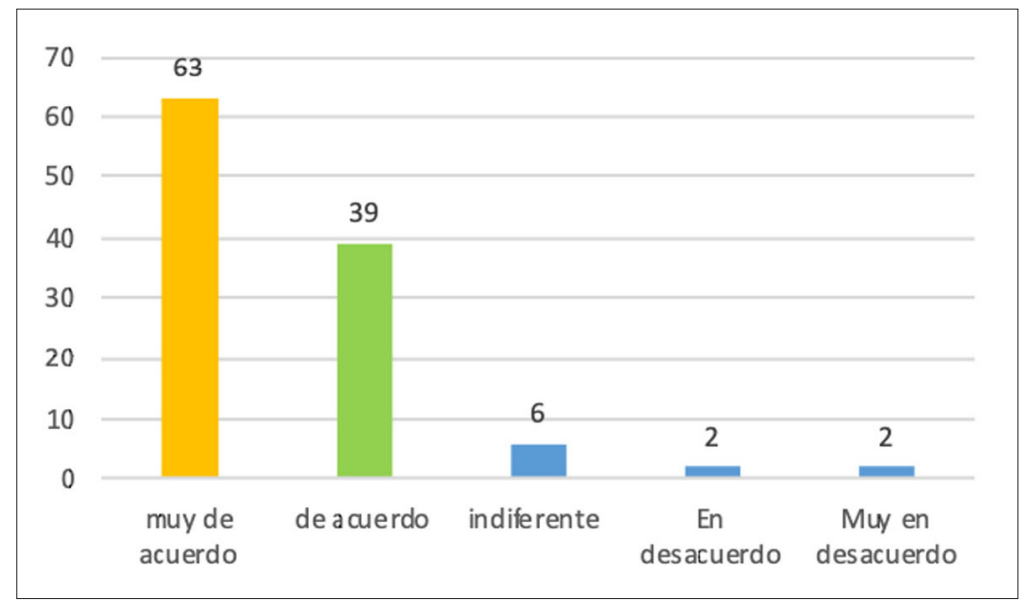

Fuente: elaboración propia (2019).

En el gráfico I se observa que un 9I\% (n=I02) de los encuestados está "muy de acuerdo" y "de acuerdo" respecto de la importancia del componente integral del ser humano, reconociendo lo corporal como una dimensión que favorece la comprensión de sí mismo, propiciando la reflexión respecto a su ser y su quehacer. Asimismo, el $5,4 \%(n=6)$ de los encuestados se muestra "indiferente" frente a esta afirmación y el 3,6\% (n=4) de los estudiantes está "en desacuerdo" y "muy en desacuerdo". 
Gráfico $\mathrm{n}^{\circ}$ 2: La posibilidad de empatizar con otro comienza cuando empatizo conmigo mismo

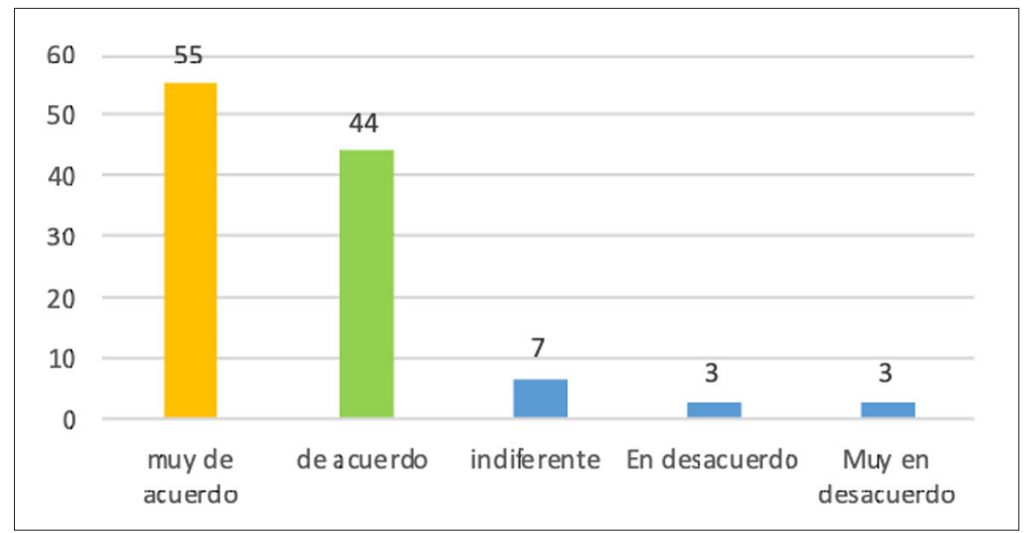

Fuente: elaboración propia (2019).

En este gráfico se muestra la valoración que realizan los estudiantes del aspecto relacional. Un 88,4\% (n=99) de los encuestados considera, a través de sus respuestas "muy de acuerdo" y "de acuerdo", que existe estrecha relación entre los propios procesos y los procesos que experimentan aquellos con quienes se relacionan. A su vez, el 6,3\% $(n=7)$ de los estudiantes se manifiesta "indiferente" y el 5,4\% $(n=6)$ están "en desacuerdo" y "muy en desacuerdo" con la afirmación anterior.

Gráfico ${ }^{\circ} 3$ : Descubro lo importante de conocerme y tomar conciencia de mí, para poder ir en ayuda de otros

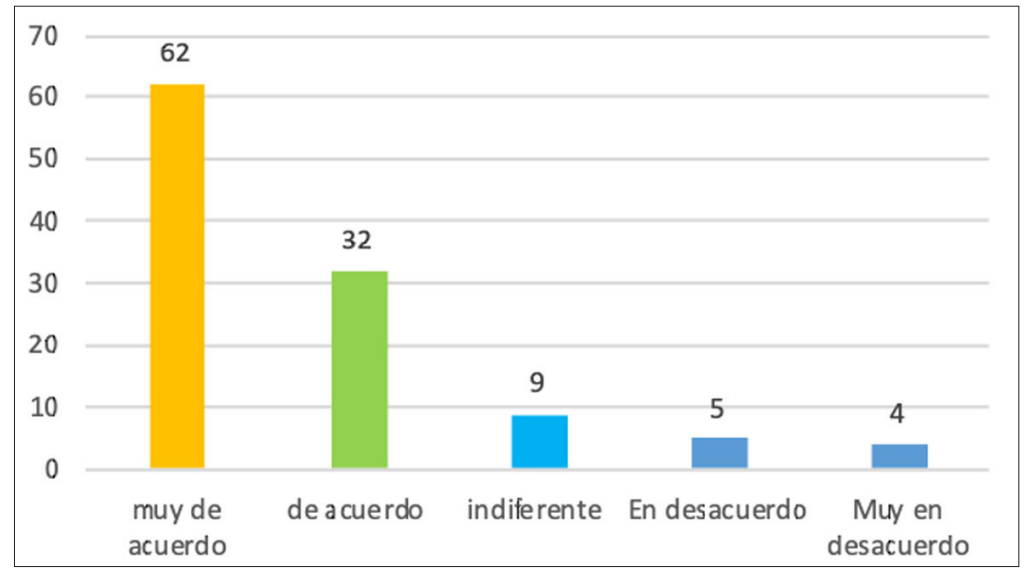

Fuente: elaboración propia (2019). 
Los resultados del gráfico 3 indican que un $84 \%(n=94)$ de los encuestados están "muy de acuerdo" y "de acuerdo", respecto de que la persona y el profesional no son elementos divisibles, aludiendo al sujeto y sus características personales como herramienta de ayuda. Destaca en este resultado que un $8 \%(n=9)$ de los estudiantes está "en desacuerdo" y "muy en desacuerdo" con esta afirmación. Existiendo al mismo tiempo un igual porcentaje de sujetos (8\%) para los cuales esta afirmación es indiferente.

Gráfico $\mathrm{n}^{\circ} 4$ : Valoro el aprender a conocerme, saber qué siento y qué puedo decidir aquí y ahora

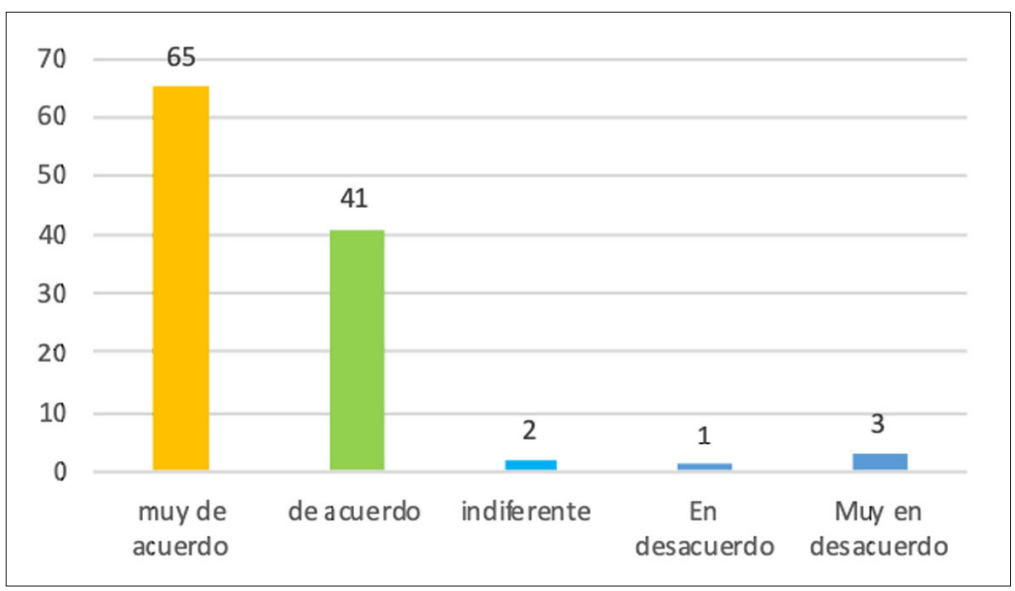

Fuente: elaboración propia (2019).

En el gráfico 4 es posible observar que en un 94,6\% $(n=106)$ de los encuestados está "muy de acuerdo" y "de acuerdo" con la valoración del componente emocional como parte esencial de la formación obtenida en esta asignatura, destacando la importancia del autoconocimiento y de vivir en el presente. Esta afirmación obtiene la más alta valoración de todas, siendo además la que presenta el menor porcentaje de estudiantes para quienes esta afirmación es indiferente, con un $1,8 \%$. $(n=2)$, y un 3,6\% $(n=4)$ de respuestas "en desacuerdo" y "muy en desacuerdo", respectivamente. 


\section{Discusión}

Es innegable que las enfermedades no solamente se relacionan con aspectos físicos o biológicos, sino que existe un componente psicosocial tremendamente valioso y que influye directamente en los procesos de salud y enfermedad. Desde esa perspectiva, si los profesionales de la salud están solamente centrados en los aspectos biomédicos, físicos o tecnológicos, no podrán generar una intervención que incluya y relacione los diversos componentes del ser humano, lo cual se constituye como una acción verdaderamente integral.

En ese contexto, la asignatura de Comunicación e Interacción Humana responde al llamado que hoy se hace a los profesionales de la salud, especialmente de enfermería, que es poner el foco en la humanización del cuidado, centrándose en trabajar pedagógicamente en el desarrollo de aquellos aspectos que respondan a ese llamado.

A partir de los resultados obtenidos en este estudio, ha sido posible aproximarse a los aprendizajes que los estudiantes de esta asignatura ban descubierto para su vida personal y profesional a partir de este modelo de enseñanza/aprendizaje llamado Comunicación e Interacción Humana, los cuales revisaremos a continuación:

Un primer aprendizaje se relaciona con la importancia de la escucba del cuerpo como una berramienta, como un lenguaje que les babla de sí mismos. De acuerdo con los resultados, existe un mayor porcentaje de estudiantes que considera relevante la perspectiva de aprender a escuchar el cuerpo y en qué lugares de su cuerpo se alojan sus emociones. La importancia del cuerpo radica, por tanto, como hemos visto en la teoría, en la necesidad de aprender a reconocerlo como un indicador, como una alarma valiosa que permita a los estudiantes tomar conciencia de las cosas que les están pasando y de las emociones que eso genera en cada uno, para, desde ahí, hacer algo con ello. Schutz propone que existe una unidad en el ser humano: el cuerpo, la mente y las emociones y que todas se relacionan y nutren entre sí (Schutz, 1973). En ese contexto es posible señalar que quizás sea el componente corporal/ emocional el que más descuidado está en el abordaje de la salud hoy en día. 
En esta línea, el desarrollo y la habilitación de los estudiantes para conocer y reconocer su cuerpo desde la mirada de las emociones, les permite reconocer también lo que a ellos mismos les produce dolor, tristeza, ansiedad o angustia, por ejemplo, pudiendo relacionarlos con componentes emocionales o psicosociales. Tal como menciona Waldow, al reconocerse como seres humanos, pueden reconocer al paciente, otro semejante, como ser humano (Waldow, 20I4), componente esencial de los cuidados humanizados.

Un segundo aprendizaje, evidenciado a partir de los resultados, se relaciona con el tópico que habla de la posibilidad de empatizar con otros, teniendo como punto de partida la empatía consigo mismo, es decir, el reconocerse. En ese contexto, la empatía consigo mismo permite al estudiante saber reconocerse desde un lugar en el mundo, desde una historia que se pone en juego en el presente, una historia que opera y se actualiza en el aquí y el ahora, por medio de diversas emociones, sensaciones, ideas y creencias, todas ellas reacciones humanas finalmente; $y$, si se facilita la posibilidad para acceder a ellas, será más factible incorporarlas en sus procesos de reflexión y en el análisis de sus propias conductas personales y profesionales. De esta manera, tendrá una posibilidad mayor de mirar más limpiamente estos procesos humanos en aquellos que serán los sujetos de su atención y cuidado, contando así con más herramientas para dar respuesta a la necesidad de las personas en los contextos de salud de sentirse escuchados, comprendidos, personas y por tanto humanos, aspectos que hoy definen la calidad de atención (Romero Massa et al., 20I6). $\mathrm{Al}$ respecto, Rowe (1999) reafirma que el aspecto terapéutico mejora en la medida en que los enfermeros se vuelven más conscientes de sí mismos.

El tercer aprendizaje que se puede extraer de las respuestas de los estudiantes se vincula con la posibilidad de descubrir la importancia de tomar conciencia de sí para poder ayudar a otros. Este aprendizaje lo consideran relevante el $84 \%$, siendo el porcentaje menor de aprobación comparado con las otras afirmaciones. Es vital que los estudiantes descubran lo importante de tomar conciencia de sí mismos para poder ir en ayuda de otros, porque no solamente reconocen a la persona, al paciente, como ser humano, sino también a sí mismos como seres humanos más 
que como profesionales de la enfermería. La toma de conciencia de sí, pilar del autoconocimiento, permite según algunos autores estar en el presente y aprender de sí mismo (Eckroth-Bucher, 2010; Scheick, 20II). Esto va en la línea mencionada por Schutz (I973), quien considera que la conciencia del cuerpo es una herramienta importante para el trabajo personal y el desarrollo emocional; generándose entonces una doble dimensión, ya que, junto con entregar herramientas para acercarse y aproximarse a la realidad de las personas con las que se trabajará, también proporciona estrategias de autocuidado, es decir, la posibilidad de poner límites, de generar mejores condiciones para sí mismo o de escucharse cuando se esté sobrepasado, pudiendo cuidarse en un contexto de trabajo en el que se convive diariamente con el dolor, el sufrimiento o la minusvalía de las personas.

No deja de llamar la atención, tal como se destaca en los resultados respecto de este tópico, que, no obstante lo dicho en el párrafo anterior, existe un $8 \%$ de los encuestados que no está de acuerdo con la afirmación planteada, presentando el mismo porcentaje de estudiantes que se muestran indiferentes. Es posible inferir que existe un grupo de estudiantes que no considera necesario o relevante la necesidad de tomar conciencia de sí mismo. Para los profesionales de la enfermería, y bajo la mirada de los cuidados humanizados que se ha ido desplegando en este documento, esta constatación puede ser explicada y mirada desde elementos socioculturales presentes en las formas y bases utilizadas para enseñar en las carreras de la salud, entre ellas Enfermería. Siguiendo a Glass y Glass (198I), estamos inmersos en una cultura de lo inmediato, que valora más los resultados que los procesos, principalmente en las esferas de la educación. Este elemento contrasta, por tanto, con la propuesta paradigmática de esta asignatura, lo cual podría explicar de alguna manera la dificultad de algunos estudiantes para aproximarse a aspectos relacionados con una mirada interna, una conciencia de sí mismos, puesto que dichos aspectos van más allá de resultados, ya que los resultados de la educación humanística no solo son difíciles de evaluar, sino que, a menudo, tardan en aparecer (Glass y Glass, I98I).

Tal como se ha expuesto desde la teoría, existe una centralidad en aspectos bio-médicos y científico-tecnológicos en la gran mayoría 
de las mallas curriculares de las carreras de Enfermería, lo cual ha ido generando una especie de alienación de estos profesionales quienes, quizás amparados en la investidura de sus uniformes, valoran más los procedimientos, lo técnico y lo verificable por sobre los aspectos internos, emocionales, de conocimiento de sí o subjetivos, haciendo cada vez más difícil un cuidado realmente humanizado que, tal como lo exponen Romero Massa, Contreras Méndez \& Mondaca Serrano (2016), no se relaciona con aspectos científicos o tecnológicos, sino que con necesidades de afecto, comprensión, protección, bienestar, ocio o información de las personas, las que son prioritarias. Además, de acuerdo con lo expresado por Maturana \& Nisis de Rezepka (I997), estas solo pueden ser desarrolladas por medio de una adecuada reflexión y conciencia de sí mismo.

Por último, un cuarto aprendizaje reflejado en el tópico presentado a los estudiantes es la valoración del saber lo que cada uno siente para aprender a conocerse y decidir en el aquí y el abora. Está afirmación es la que obtuvo mayor reconocimiento por parte de los estudiantes, entendiendo con esto que valoran la posibilidad de percibir sus emociones como un indicador importante para reconocer lo que les está pasando. Desde la perspectiva de los cuidados humanizados, se dice que la persona, al transformarse en paciente, se despersonaliza (Waldow, 20I4), por tanto, es tremendamente importante que los estudiantes valoren esta percepción de sus emociones, puesto que de esta manera podrán replicar con mayor experiencia cuando deban acercarse a una persona que se ha vuelto paciente a causa de la enfermedad, ayudándole a percibir a él o ella sus propias emociones.

El reconocimiento de las emociones no es una tarea fácil, más bien es un proceso complejo, puesto que el componente subjetivo juega un papel protagónico, y el escaso desarrollo de estas habilidades genera múltiples problemas en el trato y el abordaje de los pacientes, entre otras problemáticas. A propósito de esto, la asignatura de $\mathrm{CIH}$ se transforma en un importante espacio para el reconocimiento y desarrollo emocional, tal como menciona Rogers (I973), partiendo desde las propias emociones y las emociones de los compañeros que componen el grupo. 
Si se considera, al igual que lo hace Susana Blonch (I992), que las emociones se distribuyen en manifestaciones orgánicas, otras somáticas y unas de carácter subjetivo, siendo precisamente estas últimas en las cuales los profesionales de la salud necesitan desarrollar su acción. Es muy importante reconocer que los profesionales de la salud necesitan estar conectados con aquello que sienten, aprendiendo a reconocer lo que les produce miedo, dolor, tristeza, angustia, alegría, entre otras emociones, todo desde su particular manera de vivirlas y expresarlas, es decir, desde una dimensión totalmente subjetiva, la cual deben aprender a reconocer y valorar en cada una de aquellas personas que en el futuro serán sus pacientes.

En los procesos grupales de la asignatura es posible que los estudiantes "salgan" de aquella investidura que muchas veces entregan las carreras de la salud, dándoles la posibilidad cierta de vivir las implicancias de empatizar, más allá de lo conceptual, reconociendo a su compañero o compañera desde lo que le pasa y reconociéndolo en sí mismo. Darse cuenta de aquello les entrega la posibilidad de desplegar muchas más habilidades en estos términos y reconocer, así, cómo cada persona vive la experiencia del dolor, el miedo u otra emoción, y como eso afectará su percepción de la realidad. De este modo podrán abordar situaciones como, por ejemplo, la adhesión a los tratamientos o el reconocimiento de una enfermedad, desde el autoanálisis o la responsabilidad, permitiendo un proceso continuo de mayor eficacia para el abordaje de los procesos de salud y enfermedad.

$\mathrm{Al}$ analizar los cuatro aspectos evaluados al finalizar la asignatura, es destacable apreciar que los pilares de la psicología humanista — como el reconocimiento emocional, utilizar el cuerpo como herramienta de trabajo en la interacción con otro, la autoconciencia y el darse cuenta- son abordados. Reconocer que los estudiantes valoran estos aspectos, se transforma en una gran oportunidad para profundizar en el trabajo de humanización del cuidado desde el desarrollo de las habilidades personales y sociales, procesos que a la larga son fundamentales al momento de hacer vida la profesión.

Esos aspectos, centrales dentro de esta asignatura, entregan a los estudiantes una ventaja comparativa en la cadena de valor de los 
tratamientos y procesos que se derivan de la enfermedad. Quedando en evidencia el aporte que la asignatura de $\mathrm{CIH}$ puede hacer al proceso de humanización de la enfermería, mediante la estrategia de los grupos de encuentro.

\section{Conclusiones}

En el constante cuestionamiento de las prácticas docentes para formar profesionales que acompañen en el dolor y sufrimiento, no solo en lo técnico, sino en la gestión de cuidados humanizados, esta investigación en educación deja en evidencia que los grupos de encuentro, estrategia pedagógica utilizada para potenciar y desarrollar habilidades interpersonales y de acompañamiento emocional, contribuyen a que los estudiantes puedan reconocer sus emociones, utilizar el cuerpo como indicador de lo que les sucede en la interacción con otro, desarrollar la capacidad de autoconciencia, darse cuenta de sus propios procesos, vivenciar el presente e integrar a la persona en el profesional de enfermería, es decir, persona y profesional no son elementos divisibles.

Los resultados son muy esclarecedores. Un alto porcentaje de estudiantes valoró positivamente los contenidos y las experiencias vividas en las sesiones grupales, afirmando además que la asignatura aporta a sus proyectos de vida y refuerza la dimensión vocacional presente en la profesión de enfermería.

El enfoque humanista desarrollado en $\mathrm{CIH}$ contribuye de forma significativa a vivenciar en los futuros profesionales de enfermería que el cuidado es una cualidad humana, fortaleciendo la capacidad natural de las personas de cuidar a otros y humanizar el cuidado. Las percepciones de los propios estudiantes destacan estos aspectos, estudiantes que en la actualidad son partícipes del cuidado de las personas de su entorno y de ellos mismos, humanos cuidando a humanos.

A partir de los hallazgos obtenidos en este estudio, surgen nuevas líneas de investigación respecto a reconocer las necesidades psicoafectivas de los estudiantes al momento de iniciar la asignatura, identificar las habilidades interpersonales de mayor desarrollo en los estudiantes que 
participan en los grupos de encuentro al finalizar la asignatura, valorar la efectividad de la estrategia de "enseñanza-aprendizaje" utilizada en la asignatura de Comunicación e Interacción Humana (pre y post test) y comparar la percepción de los estudiantes que cursan la misma asignatura entre las diferentes sedes de la misma universidad, entre otras. de los estudiantes ro. cepcivas de los estudiantes al momento de iniciar la asignatura, s grupos de encuentro.

Por otro lado, esta investigación presenta el desafío de sistematizar, documentar y con eso otorgar calidad científica a los procesos humanos internos, para argumentar y proyectar un trabajo que se torna necesario e imprescindible en el escenario del cuidado humanizado en contextos de salud.

Estas experiencias, convertidas en procesos metodológicos fuertes y eficaces, pueden resultar en establecer relaciones profesionales éticamente humanas, en las que el vínculo que se funde sea nutritivo para ambas partes, profesional de la salud y paciente, y, desde esa relación, se pueda impactar en todas las dimensiones que conviven en los procesos de salud y enfermedad.

Por todo eso y más, la importancia que reflejan estos resultados es tan reveladora, puesto que, por un lado, permiten reconocer la necesidad de trabajar los aspectos internos de cada persona, $y$, por otro, visibilizan las garantías que este modelo/experiencia de $\mathrm{CIH}$ ofrece como una adecuada vía para realizar ese trabajo personal, puesto que, citando a Eduardo Galeano, "Al fin y al cabo, somos lo que hacemos para cambiar lo que somos".

\section{Referencias bibliográficas}

Aliakbari, F., Parvin, N., Heidari, M. \& Haghani, F. (2015). Learning theories application in nursing education. J Educ Health Promot., 4, 2. DOI: https:/ / doi.org/I0.4103/2277-9531.151867

Almada, H. (2015). Teoría y método en terapia Gestalt. Articulación crítica de los conceptos centrales. Ciudad de México: Pax.

Álvarez, O. \& Román de Cisneros, G. (2008). Sensibilidad. Valor condicional del Equipo de Salud para el Cuidado Humano. Revista Electronica de PortalesMedicos.Com. Recuperado de http://portalesmedicos.com/ 
publicaciones/articles/I 187/I/Sencibilidad-Valor-con-dicional-delEquipo-de-Salud-para-el-Cuidado-humano.html

Bloch, S., \& Lemeingnan. (1992). Patrones Respiratorio-Posturo-Faciales específicos relacionados con emociones básicas. Recuperado de https://docplayer. es/I3436554-Patrones-respiratorio-posturo-faciales-especificosrelacionados-con-emociones-basicas.html

Borja, G. (1995). La locura lo cura (5 ed.). Madrid: La Llave.

Capo, J. S. (1986). Psicología humanista y educacion. Anuario de Psicología, 34, 85-102.

Ceballos, P. (2010). Desde los ámbitos de enfermería, analizando el cuidado humanizado. Ciencia y Enfermería, 26(I), 3I-35. DOI: https://doi. org/I0.4067/S0717-95532010000100004

Eckroth-Bucher, M. (2010). Self-Awareness: A Review and Analysis of a Basic Nursing Concept. Advances in Nursing Science, 33(4), 297-309. DOI: https://doi.org/I0.1097/ANS.0b013e318Ifb2e4c

Egan, G. (2010). The Skilled Helper: A Problem-Management and Opportunity-Development Approach to Helping (Hse 123 Interviewing Techniques). Brooks/Cole Pub. Co. Recuperado de https://www.academia.edu/35137638/ The_Skilled_Helper

Engin, E. \& Cam, O. (2009). Effect of Self-awareness Education on the Selfefficacy and Sociotropy-Autonomy Characteristics of Nurses in a Psychiatry Clinic. Archives of Psychiatric Nursing, 23(2), I48-I56. DOI: https://doi.org/IO.I0I6/j.apnu.2008.05.003

Guey, C., Cheng, Y. \& Shibata, S. (2010). A triarchal instruction model: Integration of principles from Behaviorism, Cognitivism, and Humanism. Procedia - Social and Behavioral Sciences, 9, I05-I18. DOI: https://doi.org/I0.1016/j.sbspro.2010.12.122

Jack, K. \& Smith, A. (2007). Promoting self-awareness in nurses to improve nursing practice. Nursing Standard, 2l(32), 47-52. DOI: https://doi. org/10.7748/ns2007.04.21.32.47.c4497

Kepner, J. (I999). Proceso corporal. Un enfoque gestalt para el trabajo corporal en psicoterapia. Manual Moderno.

Landman Navarro, A., Del Alcázar Pabst, R. M., Madrid Zamorano, Y., Pais Veliz, L., Rosenkranz Moreno, E. \& Vivanco Lobato, I. (2016). Habilidades para el cuidado humanizado de estudiantes de enfermería. Enfermería: Cuidados Humanizados, 5(I), 29. DOI: https:// doi.org/I0.22235/ech.v5iI.I I90 
Maslow, A. (1968). Some Educational Implications of the Humanistic Psychologies. Harvard Educational Review, 38(4), 685-696. DOI: https:/ / doi.org/IO.I7763/haer.38.4.j07288786v86w660

Maturana, H. R. (200I). Emociones y lenguaje en educación y política ( $8^{\mathrm{a}} \mathrm{ed}$.). Santiago de Chile: J.C. Sáez.

Maturana, H. R. \& Nisis de Rezepka, S. (1997). Formación bumana y capacitación $\left(2^{\mathrm{a}}\right.$ ed). Santiago de Chile: Dolmen Ediciones.

Organización Mundial de la Salud. (20I4). Documentos básicos. Recuperado de https://apps.who.int/gb/bd/PDF/bd48/basic-documents-48thedition-sp.pdf

Parveen, S. (2015). Self-Awareness as a Therapeutic Tool for Nurse/Client Relationship. International Journal of Caring Sciencies, 8(I), 2I I-2I6.

Poblete Troncoso, M. \& Valenzuela Suazo, S. (2007). Cuidado humanizado: Un desafio para las enfermeras en los servicios hospitalarios. Acta Paulista de Enfermagem, 20(4), 499-503. DOI: https://doi.org/I0.1590/S0I0321002007000400019

Ramos Guajardo, S. \& Ceballos Vásquez, P. (2018). Cuidado humanizado y riesgos psicosociales: Una relación percibida por profesionales de enfermería en Chile. Enfermería: Cuidados Humanizados, 7(I). DOI: https:// doi.org/I0.22235/ech.v7iI.I537

Rasheed, S. P., Younas, A. \& Sundus, A. (2019). Self-awareness in nursing: A scoping review. Journal of Clinical Nursing, 28(5-6), 762-774. DOI: https://doi.org/IO.IIII/jocn.I4708

Rogers, C. (I969). Freedom to learn: A view of what education might become. Columbus, Ohio: C. E. Merrill Pub. Co.

Rogers, C. (1973). Grupos de Encuentro. Buenos Aires: Amorrortu Editores.

Rogers, C. (2007). The necessary and sufficient conditions of therapeutic personality change. Psychotherapy: Theory, Research, Practice, Training, 44(3), 240-248. DOI: https://doi.org/IO.I037/0033-3204.44.3.240

Romero Massa, E., Contreras Méndez, I. M. \& Mondaca Serrano, A. (20I6). Relación entre cuidado humanizado por enfermería con la hospitalización de pacientes. Hacia La Promoción de La Salud, OI2 I-7577(2462-8425), 2636. DOI: https://doi.org/I0.I7I5I/hpsal.20I6.2I.I.3

Rowe, J. (1999). Self-awareness: Improving nurse-client interactions. Nursing Standard, 14(8), 37-40. DOI: https://doi.org/I0.7748/ ns 1999.II.I4.8.37.c2709

Scheick, D. M. (20II). Developing Self-Aware Mindfulness to Manage 
Countertransference in the Nurse-Client Relationship: An Evaluation and Developmental Study. Journal of Professional Nursing, 27(2), I I4-I23. DOI: https://doi.org/I0.10I6/j.profnurs.2010.10.005

Schutz, W. (1973). Todos somos uno. La cultura de los encuentros. España: Amorrortu Editores.

Stein, K. (1995). Schema Model of the Self-Concept. Journal of Nursing Scholarship, 3(27), I87-193.

Waldow, V. R. (2014). Cuidado humano: La vulnerabilidad del ser enfermo y su dimensión de trascendencia. Index de Enfermería, 23(4), 234-238. DOI: https://doi.org/I0.432I/SI 132-I2962014000300009

Walter, R., Davis, K. \& Glass, N. (I999). Discovery of self: Exploring, interconnecting and integrating self (concept) and nursing. Collegian, 6(2), I2-I5. DOI: https://doi.org/I0.10I6/SI322-7696(08)60324-8 